\title{
Magnetic resonance imaging and Ménière's disease—unavoidable alliance
}

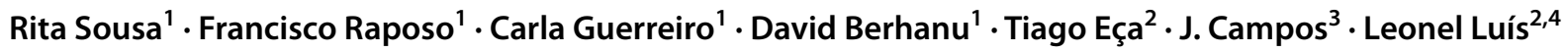

Received: 7 September 2020 / Accepted: 31 May 2021

(c) The Author(s), under exclusive licence to Springer-Verlag GmbH Germany, part of Springer Nature 2021

\begin{abstract}
Ménière's disease (MD) is a clinical syndrome characterized by recurrent episodes of spontaneous vertigo, unilateral fluctuating sensorineural hearing loss, tinnitus, and aural fullness. Endolymphatic hydrops is recognized as the pathophysiological substrate of the disease, having been demonstrated in anatomical pathological studies and more recently by magnetic resonance imaging (MRI). The current criteria of the disease, however, remain symptom based and do not include the demonstration of endolymphatic hydrops. The authors review MRI techniques and diagnostic criteria of endolymphatic hydrops and the role of MRI in MD is discussed.
\end{abstract}

Keywords Endolymphatic hydrops · Menière's disease · Magnetic resonance imaging $\cdot$ Hydropic Ear $\cdot$ Imaging inner ear

\section{Introduction-current issue}

MD is a chronic disease with a prevalence of $200-500$ per 100,000 individuals [1], characterized by a recurrent clinical syndrome of audiovestibular symptoms, namely, spontaneous vertigo, unilateral hearing loss, aural fullness, and tinnitus.

In 1861, Prosper Ménière was the first to recognize the inner ear at the origin of the symptoms with endolymphatic hydrops only later, in 1937, being described by British [2] and Japanese [3] researchers. Nowadays, its cause remains undetermined, but the pathophysiological substrate is known: the increase in the endolymphatic space of the membranous labyrinth, partially occupying the usual space of the perilymph (Fig. 1).

Although imaging evolution currently allows the identification and quantification of endolymphatic hydrops, the

Rita Sousa

ritafsousa1@gmail.com

1 Neuroradiology Department, Centro Hospitalar Universitário Lisboa Norte, Rua Casal da Raposa, 46, Caselas, 1400-050 Lisbon, Portugal

2 Otorhinolaryngology Department, Centro Hospitalar Universitário Lisboa Norte, Lisbon, Portugal

3 Imagiology Department, Lisbon Red Cross Hospital, Lisbon, Portugal

4 Institute of Molecular Medicine, Lisbon, Portugal diagnostic criteria remain clinical and based on symptoms and hearing evaluation only, and do not consider the vestibular evaluation or the demonstration of endolymphatic hydrops.

\section{Clinical diagnostic criteria of MD}

The clinical criteria for diagnosing MD have undergone some evolution over time, although they have maintained a classification based on symptoms, which are often difficult to target. In 1967, Hinchcliffe [4] described the classic clinical picture, with a triad of spontaneous vertigo, hearing loss, and aural fullness/tinnitus, after excluding other causes (pathology of the middle ear, central nervous system, pharmacology, systemic diseases, among others); later on, some light forms of the disease were described, in which the clinical picture is not complete [5].

In 1972, the American Academy of Otolaryngology and Head and Neck Surgery adopted the nomenclature of "vestibular MD" and "cochlear MD" [6], abandoning it repeatedly in 1985 [7] and in 1995 [8], alleging insufficient evidence that these monosymptomatic conditions have a common pathophysiology to MD.

The criteria revised in 1995 classify MD as "possible MD"-episodic vertigo or fluctuating deafness, "probable MD"-a 20-min rotating vertigo episode accompanied by tinnitus and documented sensorineural hearing 


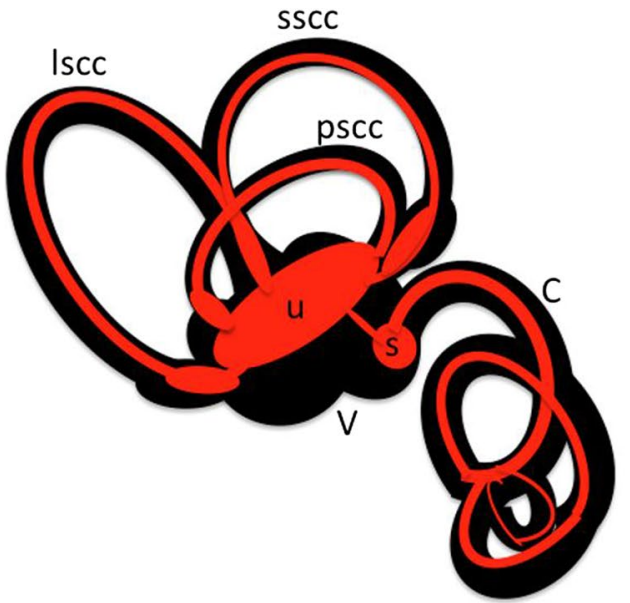

Fig. 1 Schematic representation of the membranous labyrinth with normal sized endolymphatic space on the left (healthy ear) and enlarged endolymphatic space on the right (MD). Endolymphatic space is represented in red and perilymphatic space is black. Utricle (u) is in close anatomic relation with semicircular canals (lateral, lscc; superior, sscc; posterior, pscc). Saccule (s) is located in ante-

loss, "definite MD"- two or more episodes of vertigo of 20 or more minutes in duration, accompanied by tinnitus and documented sensorineural hearing loss, and "certain MD"-criteria for definitive MD and additional histological, usually post-mortem, demonstration of hydrops in the affected ear. This classification continues to include the diagnosis of MD in patients whose installation of the complete clinical picture is more indolent (20\% may take up to 5 years and $10 \%$ more than 10 years to present the full clinical picture) [9].

In 2015, the diagnostic MD criteria were reclassified by Barany Society [10] (Table 1) as "definite MD" and "probable MD," but here removing the "certain MD" that included the anatomopathological demonstration endolymphatic hydrops. According to the 2015 consensus, the "definite MD"consists of two or more episodes with $20 \mathrm{~min}$ to $12 \mathrm{~h}$ of vertigo associated with unilateral sensorineural hypoacusia for low to medium frequencies and fluctuating aural symptoms, such as tinnitus and ear fullness with "probable MD" not including the audiometric demonstration for hearing loss.

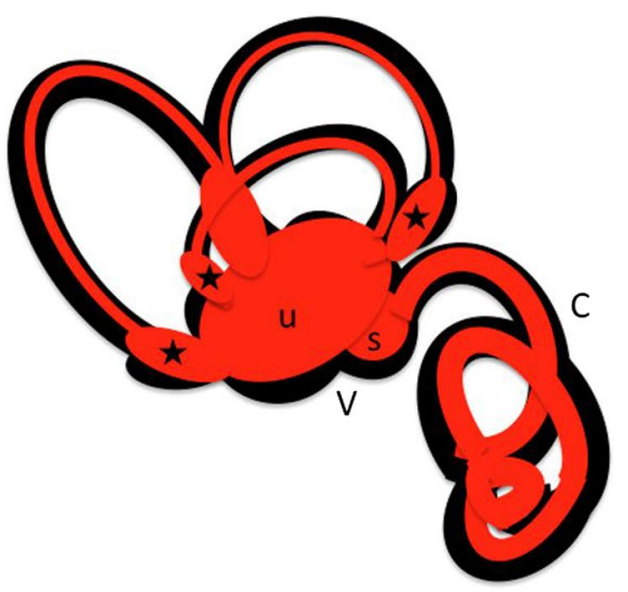

rior, inferior, and medial part of vestibule $(\mathrm{V})$ (pars inferior) in anatomic proximity to cochlea (C). In endolymphatic hydrops (right), the endolymphatic spaces enlarge (red); utricle and saccule are enlarged, partly obliterating perilymphatic space and herniating into the ampullary $(\star)$ and non ampullary ends of semicircular canals

This classification has been contested mainly because it excludes endolymphatic hydrops demonstration from the criteria, especially at a time when it is possible to demonstrate it in vivo, by MRI. In 2016, Nakashima [11] argued that all patients with clinical suspicion of MD should be studied with MRI of the inner ear, proposing a new classification of MD including MRI of endolymphatic hydrops. Bearing in mind that the installation of the "complete" clinical picture may take more than 10 years in many cases [9], the authors [11] propose that monosymptomatic patients with endolymphatic hydrops should be treated as having MD, as suggested by the 1972 classification, in which MD subtypes were recognized without the complete clinical picture ("vestibular MD" and "cochlear MD").

Based on the diversity of clinical presentations and with an emphasis on the importance of hydrops diagnosis, Gurkov goes further and demands not only new diagnostic criteria (including endolymphatic hydrops) [12] but also a new stratification [13], in patients "with hydrops" (where MD would be included) and patients "without hydrops," as a way to select those who would benefit from therapy
Table 1 Barany Society diagnostic criteria of MD proposed in 2015 [10]

\begin{tabular}{ll}
\hline Definite MD & - At least two episodes of spontaneous vertigo that last 20 min to $12 \mathrm{~h}$ \\
- Audiometric documented low to medium frequency neurosensorial hearing loss in \\
the affected ear, before, during, or after the vertigo episode \\
- Cochlear fluctuating symptoms (hearing, aural fullness, tinnitus) in the affected ear \\
- No other diagnosis of vestibular pathology \\
Probable MD & - At least two episodes of vertigo or dizziness each lasting 20 min to $24 \mathrm{~h}$ \\
& - Cochlear fluctuating symptoms (hearing, aural fullness, tinnitus) in the affected ear \\
& - No other diagnosis of vestibular pathology
\end{tabular}


directed at hydrops in the evolution of the natural history of the disease. In fact, it seems endolymphatic hydrops is not exclusive of typical MD, and it can be found in atypical MD presentations (such as monosymptomatic cases) and in other conditions, such as malformations, intra labyrinthine tumors, and endolymphatic sac tumors [14].

\section{Anatomopathological evidence of endolymphatic hydrops in MD}

Endolymphatic hydrops is the most original finding in MD (Fig. 1) and has always been considered in discussions of the disease. In 2013, Foster et al. [15] reviewed all published anatomopathological studies of temporal bones that included 165 patients with clinical suspicion of MD according to 1995 [8] clinical criteria, having found endolymphatic hydrops in all 163 of the cases of definitive MD in at least one ear (98.8\%). It was not possible to know whether the two cases without endolymphatic hydrops would have developed criteria for definite DM due to the lack of clinical records. The authors concluded that endolymphatic hydrops is more than an epiphenomenon, since there is a practically perfect association between each case of definitive MD and the occurrence of endolymphatic hydrops. They theorize that endolymphatic hydrops probably will not be enough to cause the complete clinical picture, but associated with one or more additional (unknown) cofactors, asymptomatic endolymphatic hydrops may be converted into symptomatic MD.

\section{MRI of endolymphatic hydrops in humans}

Until recently, the demonstration of endolymphatic hydrops was not likely to be included in the criteria for diagnosing the disease in vivo, since its demonstration was limited to anatomopathology [16], so the diagnostic criteria were mainly clinical. In this era, the role of MRI was limited to excluding other causes for the clinical picture, such as cerebrospinal fluid hypotension, cerebrovascular disease, inflammatory conditions (such as multiple sclerosis), arteriovenous malformations, posterior fossa occupying space lesions, and inner ear pathology (malformative, inflammatory and tumor). Computed tomography allowed, in a complementary way to MRI, the detailed evaluation of the middle ear and the bony labyrinth, allowing the identification of inflammatory pathology, otosclerosis, as well as some findings that are believed to be associated with MD, namely, stenosis of the vestibular aqueduct, high riding jugular bulb, and dehiscences of the semicircular canals [17].

In 2005, Zou et al. [18] managed to separate the endo and perilymphatic compartments by MRI in animal and human models, and 2 years later, the Japanese group published on the demonstration of endolymphatic hydrops by MRI in humans $[19,20]$ through specific algorithms with FLAIR (Fluid Attenuation Inversion Recovery) sequences. The latter, more sensitive in the identification of contrast in the perilymph, when compared to the T1 sequence, permits visualizing very small amounts of contrast in the inner ear. This was a crucial milestone, since it allows diagnosing endolymphatic hydrops in vivo, corroborating the clinical diagnosis in MD patients. The basis of the technique explores the different penetration capabilities of the contrast agent in the endo and perilymphatic spaces, obtaining a higher signal in the perilymph, which can thus be distinguished from the endolymph. Later, the same group managed to improve the contrast between the endolymph (without contrast) and the bone, through the turbo-spin echo 3D inversion-recovery technique (3D-Real-IR) [21]. This allowed to better diagnosing endolymphatic hydrops in definitive MD and in monosymptomatic cases, as well as to assess their evolution over the course of the disease [22]. Endolymphatic hydrops is present in all patients with definitive MD [23, 24], as it has been demonstrated with the anatomopathological studies previously mentioned. Also, it is now known that the type of symptoms present is related to the different locations (utricle, saccule, cochlea) preferentially affected by hydrops [9], although there is no direct and simple relationship between clinical manifestations and the degree and topography of the hydrops for each individual, with some patients showing marked endolymphatic hydrops with relatively preserved auditory function. On the other hand, it is also possible not only to identify endolymphatic hydrops in the ears that are still asymptomatic [9] but also to identify bilateral hydrops in more advanced clinical stages of the disease [25].

The literature on MRI for the diagnosis of endolymphatic hydrops in MD is still globally scarce and difficult to dissect due to the great variability in patient selection (subjective clinical criteria), the absence of healthy control groups (it is difficult to obtain healthy controls, due to ethical concerns due to the need to inject contrast with some studies establishing the "asymptomatic" ear as the control, problematic approach due to the usual bilateral nature of this condition), the variability of the used MR techniques (difficult to reproduce in different devices), the analysis of the images obtained (qualitative, semi-quantitative and quantitative; pre- or post-processed images), the different scales for presenting the results (use of different cut-off values for endolymphatic hydrops and use of different measure methods, for example, including or not the ampulla of semicircular canals), and the lack of longitudinal studies necessary to clarify the benefit of MRI in the diagnosis and follow-up of these patients. 


\section{Intratympanic and intravenous gadolinium administration techniques}

In 2007, Nakashima et al. [19] published the first in vivo demonstration of endolymphatic hydrops in MD, after intratympanic contrast injection. For this technique, a diluted gadolinium suspension $(0.3-0.6 \mathrm{ml}$ with $1: 8$ dilution) is injected through the tympanic membrane under microscopic control. This contrast is absorbed mainly through the oval and round window membranes to the perilymphatic space of that ear, not diffusing to the endolymphatic space due to the presence of tight junctions (Fig. 2). After $24 \mathrm{~h}$, MRI images are acquired in a high magnetic field device (3 T) with the T2 3D FLAIR sequence, showing the hyperintense perilymphatic space, which contrasts with the hypointense endolymphatic space (Fig. 2).

When compared with the intravenous technique, this method allows higher concentrations of contrast in the perilymph, making the interpretation of the images easier. Nevertheless, the asymmetry in the distribution of the contrast may occur for reasons unrelated to the perilymphatic volume, such as different contrast permeability of the oval and round windows. Also, due to the contrast distribution pattern, it has greater expression in the cochlear basal loop. Overall, it is a more invasive technique, but with a lower risk of systemic toxicity, although local toxicity has rarely been described [26]. Several publications demonstrated the safety of the intratympanic use of gadolinium in diluted solutions [27-29] but the intratympanic use of gadolinium is still an off label use of gadolinium, contrary to the intravenous route of administration. Importantly, the evaluation of the two ears is independent. As some patients are reluctant to intratympanic injection of the "asymptomatic" ear, some authors combine the intratympanic (on the symptomatic side) and intravenous (to assess the "asymptomatic" ear) techniques [30].

In 2010, Naganawa [31] described the intravenous technique of visualizing the endolymphatic and perilymphatic spaces by MRI through the intravenous administration of a simple dose of gadolinium $(0.1-0.2 \mathrm{ml} / \mathrm{kg})$. Less invasive and with a more homogeneous contrast distribution, this allows for the evaluation of changes in the permeability of the blood-perilymphatic barrier, unable to assess with the intratympanic technique. This technique also requires a shorter 4-h interval between intravenous administration of contrast and image acquisition (Fig. 2).

Several publications used these techniques (intravenous, intratympanic or combined), with variable contrast doses and different technical parameters, such as the number of receptor channels of the skull antenna. Both techniques (intra-tympanic and intravenous) with its advantages and

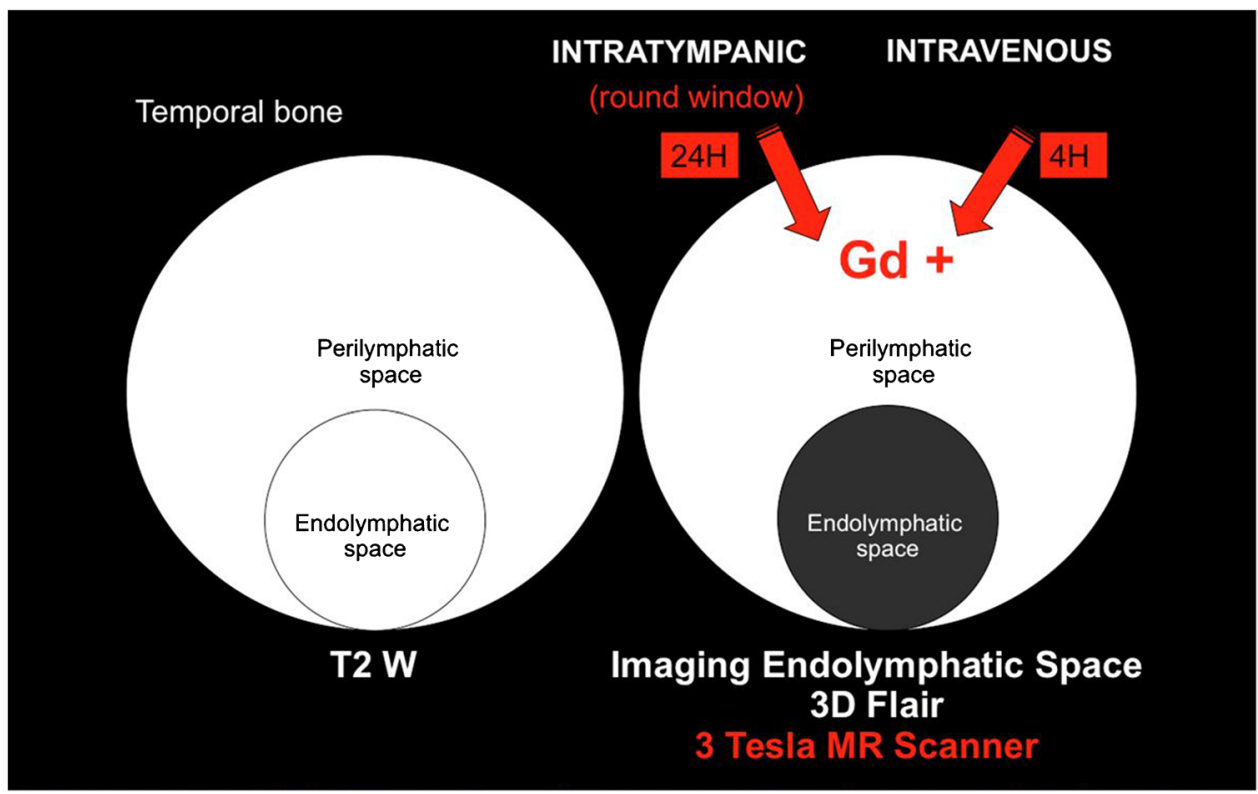

Fig. 2 Schematic representation of the endolymphatic and perilymphatic spaces by magnetic resonance. Left image-in the "conventional" T2 3D sequence, the contents of the membranous labyrinth (perilymphatic and endolymphatic spaces) are both hyperintense. Right image-when contrast (intravenous or intratympanic) is administered, it will reach the perilymphatic space (with elevation of the signal) but does not pass into the endolymphatic space; thus, the endolymphatic space presents suppression of the signal in $3 \mathrm{D}$ FLAIR (becoming hypointense) while the perilymphatic space is not suppressed in this sequence (hyperintense). It is difficult to establish the boundary between the endolymphatic space and the bone (both hypointense) 
disadvantages show consistent correlations between the degree of hydrops and the result of audiovestibular tests (audiometry, electrocochleography and caloric tests), especially with pure tone audiometry [32].

A recent paper from Gurkov [33] focused on the "index ear" concept. It is defined as the more symptomatic ear based on patient history, low frequency audiometric data, and caloric testing. He finds a strong correlation between the more symptomatic side and the more hydropic side, and highlights that unilateral imaging (intratympanic technique with higher imaging quality) might be sufficient in most patients for the diagnosis of endolymphatic hydrops.

In 2017, MR techniques without contrast administration were described [34] with T2 3D images (FIESTA, CISS, DRIVE), allowing for the measurement of the diameter of the saccule. The limitations in cochlear evaluation and the reliability of the small structures measurements were a matter of debate among some authors [35].

\section{MRI sequence parameters and post-processing}

The emergence of the intravenous gadolinium technique has added information regarding the permeability of the bloodperilymphatic barrier, but with the disadvantage of lower gadolinium concentrations in the perilymph. This motivated the development of techniques for detecting the faintest signs of contrast in the perilymph [36, 37] by adjusting the inversion time between the null point of the perilymph with gadolinium and the endolymph without gadolinium. This technique uses subtraction of images with different inversion times, allowing a good visualization of the endolymphatic, perilymphatic, and bone spaces in a single image (Fig. 3). This process is called HYDROPS (HYbriD of Reversed image Of Positive endolymph signal and native image of positive perilymph Signal) (subtraction between PPI (positive perilymph) and PEI (positive endolymph) [36]. HYDROPS2 is reconstructed by subtracting the cisternoMR (usually T2 3D turbo spin echo with variable flip angle).

The acquisition times were, however, initially long (initially over $30 \mathrm{~min}$ ), which limited its routine application. They then developed the HYDROPS2-Mi2 method (HYbrid of Reversed image Of MR cisternography and a positive Perilymph Signal by heavily T2-weighted 3D-FLAIRMultiplied by T2), reducing the acquisition time by twothirds [38]. This represents HYDROPS and HYDROPS2 multiplied by the cisternoMR image in order to increase the signal-to-noise ratio (obtaining HYDROPS-Mi2 and HYDROPS2-Mi2 respectively).

Some authors defend the use of T2 3D FLAIR maximum intensity projection reconstruction (MIP) images (comparing to two-dimensional evaluations) for the evaluation of endolymphatic hydrops [39].

Indicative parameters of the T2 FLAIR sequence are widely available in literature [31, 40], but have to be optimized for each device and images should be obtained in asymptomatic control groups. An input from an MRI

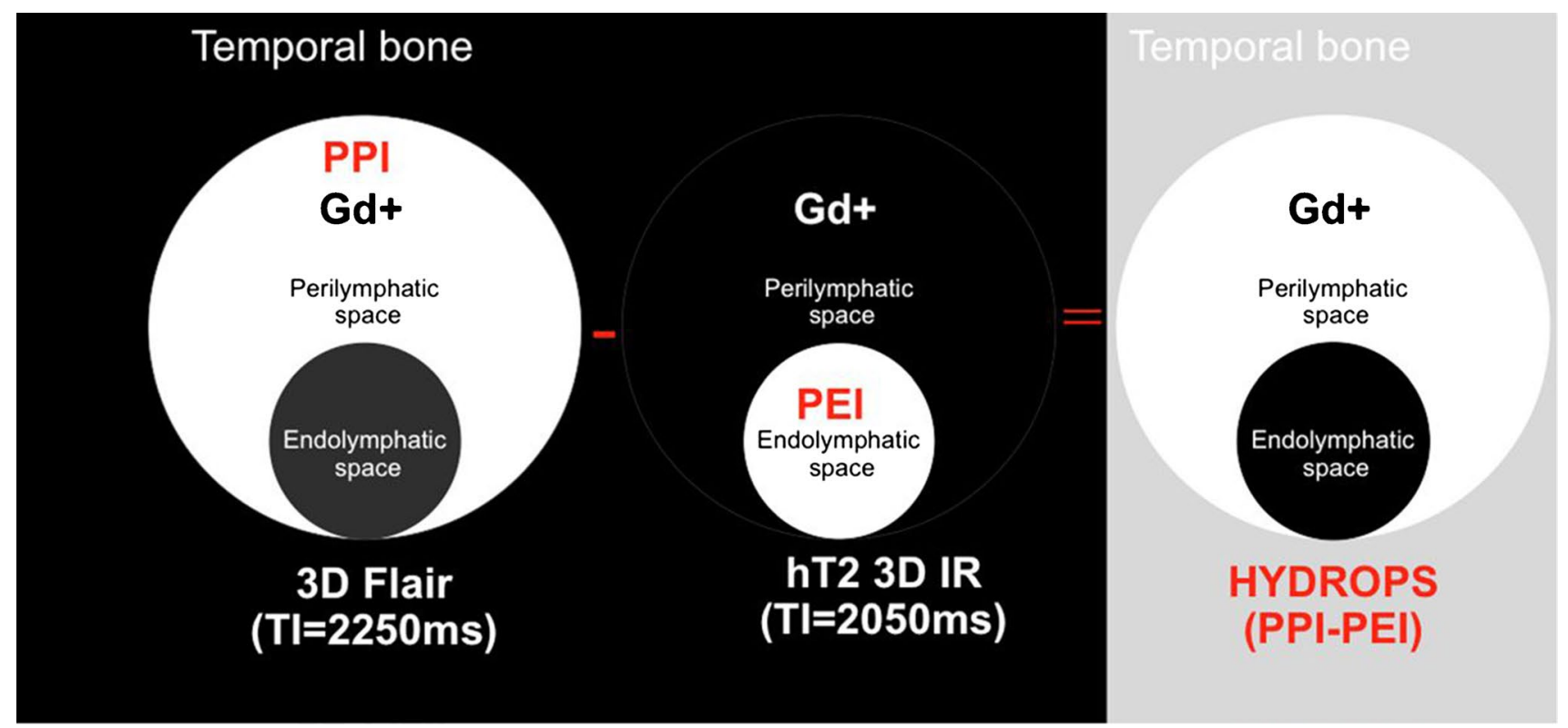

Fig. 3 Schematic representation of the HYDROPS (hybrid of the reversed image of the positive endolymph signal and native image of the positive perilymph signal) image - the technique uses the subtraction of images with different inversion times, namely from the T2
3D IR image (PEI positive endolymphatic image) to the 3D flair (PPI positive perilymphatic image), improving the interface between the 3 spaces in a single image: endolymphatic (hypointense), perilymphatic (hyperintense), and bone (neutral) 
physicist is ideal, although not always feasible. The sequence parameters such as the echo time (TE) as great impact on the degree of enhancement [31] and variations in the inversion time (TI) may influence the apparent size of endolymphatic structures [41]. Increased signal from enhancing perilymph can be obtained with a constant flip angle. Isotropic sequences should be used given the need to evaluate multiple planes [42], but a better definition of cochlear hydrops implicates improving in-plane resolution at expense of isotropic imaging (avoiding EH being repeated). A voxel size of $0.7 \mathrm{~mm}$ contributes to an adequate signal to noise ratio [40]. The endolymphatic hydrops sequence should be performed 3.5 to $4.5 \mathrm{~h}$ after intravenous gadolinium injection [43]. Some centers use double dose of intravenous contrast ( $0.2 \mathrm{~mm} / \mathrm{kg}$ body weight) [40] but single dose is feasible by using more heavily T2-weighted FLAIR sequences [31].

In addition to endolymphatic hydrops imaging, it is necessary to exclude other differential diagnosis that can present with audiovestibular symptoms (Table 3). For this reason, we usually use axial T2 Flair and diffusion imaging of the brain (to exclude intra axial lesion) and high resolution T1-weighted imaging (pre- and postgadolinium) and T2-3D weighted imaging of the inner ear/cerebellar pontine angle (to exclude space occupying lesions and evaluate the signal and morphology of inner ears structures).

\section{MRI antenna and image quality}

Though first reports of in vivo endolymphatic hydrops have been made at 3 T MRI [19], and grading endolymphatic systems have been developed from 3 T MRI (Figs. 4 and 5) it is also possible to obtain reliable information from 1.5 T MRI. Endolymphatic hydrops was demonstrated in humans for the time on a 1.5 T MRI in 2012 [44]. Using phase sensitive inversion recovery MRI following intratympanic administration of gadolinium and multiple TI values, the authors obtained confident identification of endolymphatic hydrops in Meniere's patients even when perilymph opacification was suboptimal at one TI value. One year after, Naganawa demonstrated endolymphatic hydrops in Ménière's disease using intravenous administration of single dose of gad in $1.5 \mathrm{~T}$ scanner and a 32-channel head coil [45], optimizing the protocol with enlargement of pixel size to compensate the decrease in signal-to-noise ratio. Recently, Gurkov [46] introduced a $1.5 \mathrm{~T}$ high-resolution MRI protocol with intratympanic technique that bypasses the need for $3 \mathrm{~T}$ scanners and permits endolymphatic hydrops imaging across different health care systems around the world. He proposes a two slice system for grading vestibular hydrops.

As the inner ear is an anatomical structure with a region of interest of small dimensions and low signal, factors such as sensitivity and pixel resolution to obtain images with clinical utility become critical in magnetic resonance. Recently, Zou et al. [47] reviewed MRI studies using different MR coil. Regardless of the $3 \mathrm{~T}$ magnetic resonance model and the sequences used, coils with a greater number of elements produce images with better spatial resolution. However, in $3 \mathrm{~T}$, is it possible to obtain fair images of endolymphatic inner ear spaces with lower number of elements-images from Figs. 6, 7, 8, 9, 10, 11, 12 and 13 were obtained from a 8-channel head coil from Philips Achieva 3 Tesla MRI scanner, using intravenous single dose of gadolinium and a delayed acquisition $4 \mathrm{~h}$ after contrast injection.

The sensitivity of magnetic resonance is dependent on the type of radio frequency coil used to receive the signal. With the simultaneous acquisition and the obtaining of combined data from several receivers positioned

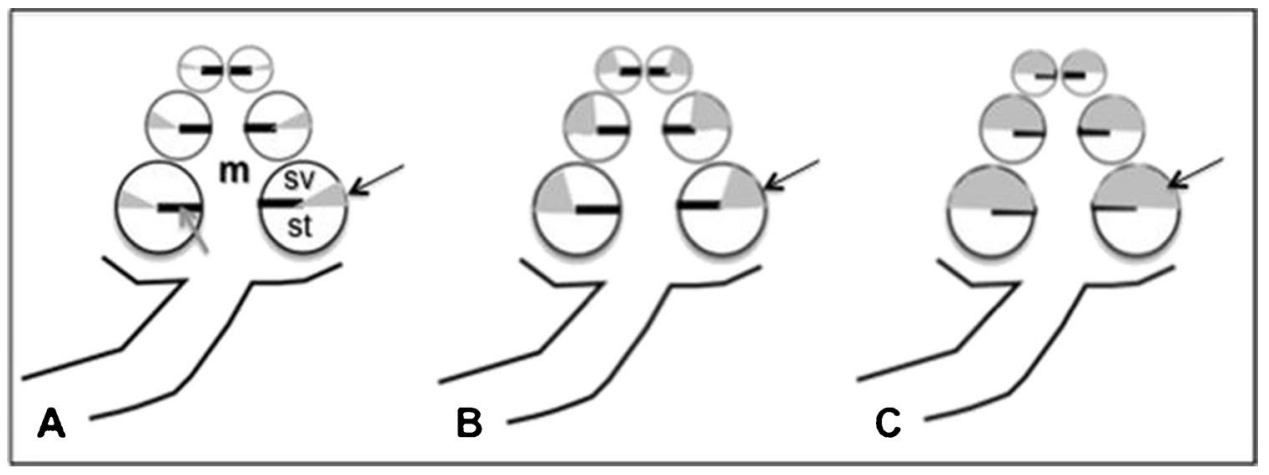

Fig. 4 Schematic representation of the cochlear membranous labyrinth in the axial plane along the greater axis of modiolus (m), according to Nakashima, Barath and Bernaerts. A-Grade 0 cochlear membranous labyrinth. Bony spiral lamina (grey arrow), scala vestibuli (sv), scala tympani (st), and small scala media (black arrow).
B-Grade 1 cochlear endolymphatic hydrops with scala media enlargement (black arrow) and partial obliteration of scala vestibuli. C-Grade 2 cochlear endolymphatic hydrops with total obliteration of scala vestibuli by enlarged endolymphatic space (solid arrow) 


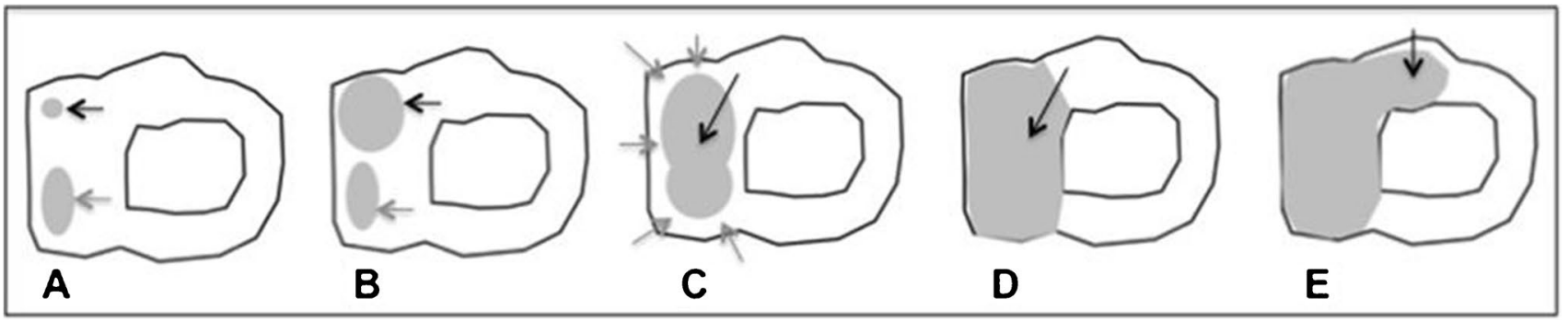

Fig. 5 Schematic representation of the vestibular membranous labyrinth in the axial plane, A to D according Nakashima [47] (A, C, D), Barath [51] (A, C, D), Bernaerts (A, B, C, D) [49], adding a fourth degree (E) from Gurkov [16]. A-Grade 0 (normal) vestibular membranous labyrinth. Small sacule (black arrow) and utricle (grey arrow), usualy visible at pars inferior. B-Grade 1 (mild) vestibular endolymphatic hydrops with sacule enlargement (black arrow) but still separated from the utricle (grey arrow). $\mathbf{C}$-Grade 2 (moderate) endolymphatic hydrops with confluence of utricle and sacule (black arrow) and partial obliteration of perilymphatic space (grey arrows). D-Grade 3 (severe) vestibular endolymphatic hydrops with marked enlargement of the confluent endolymphatic space (black arrow) and total obliteration of perilymphatic space of the vestibule. $\mathbf{E}$ - Grade 4 (extreme) vestibular endolymphatic hydrops with herniation into the ampullae of lateral semicircular canal (black arrow)
Fig. 6 Patient with a single episode of spontaneous vertigo for 2 years and bilateral low frequency neurosensorial earing loss, more severe on the left. a and $\mathbf{b}$ Axial T2 3D images of the right (a) and left (b) ears: it is possible to evaluate the morphology and dimensions of the total membranous labyrinth (vestibules - white arrowsand cochleas). $\mathbf{c}$ and $\mathbf{d}$ Axial HYDROPS images of the right (c) and left (d) ears obtained $4 \mathrm{~h}$ after intravenous gadolinium injection: endolymphatic space is enlarged in the left ear (d) - increase in scala media of apical (black arrow) and middle turn (grey arrow) of cochlea, completely obliterating the scala vestibuli space (cochlear hydrops grade 2); sacule and utricle confluence (white arrow) completely effacing perilymphatic space (vestibular hydrops grade 3); in the right ear (c), the endolymphatic space is reduced (normal in size) in apical turn (black arrow) and middle turns (grey arrow) of cochlea; sacule (solid white arrow) is smaller than utricle (dashed white arrow)

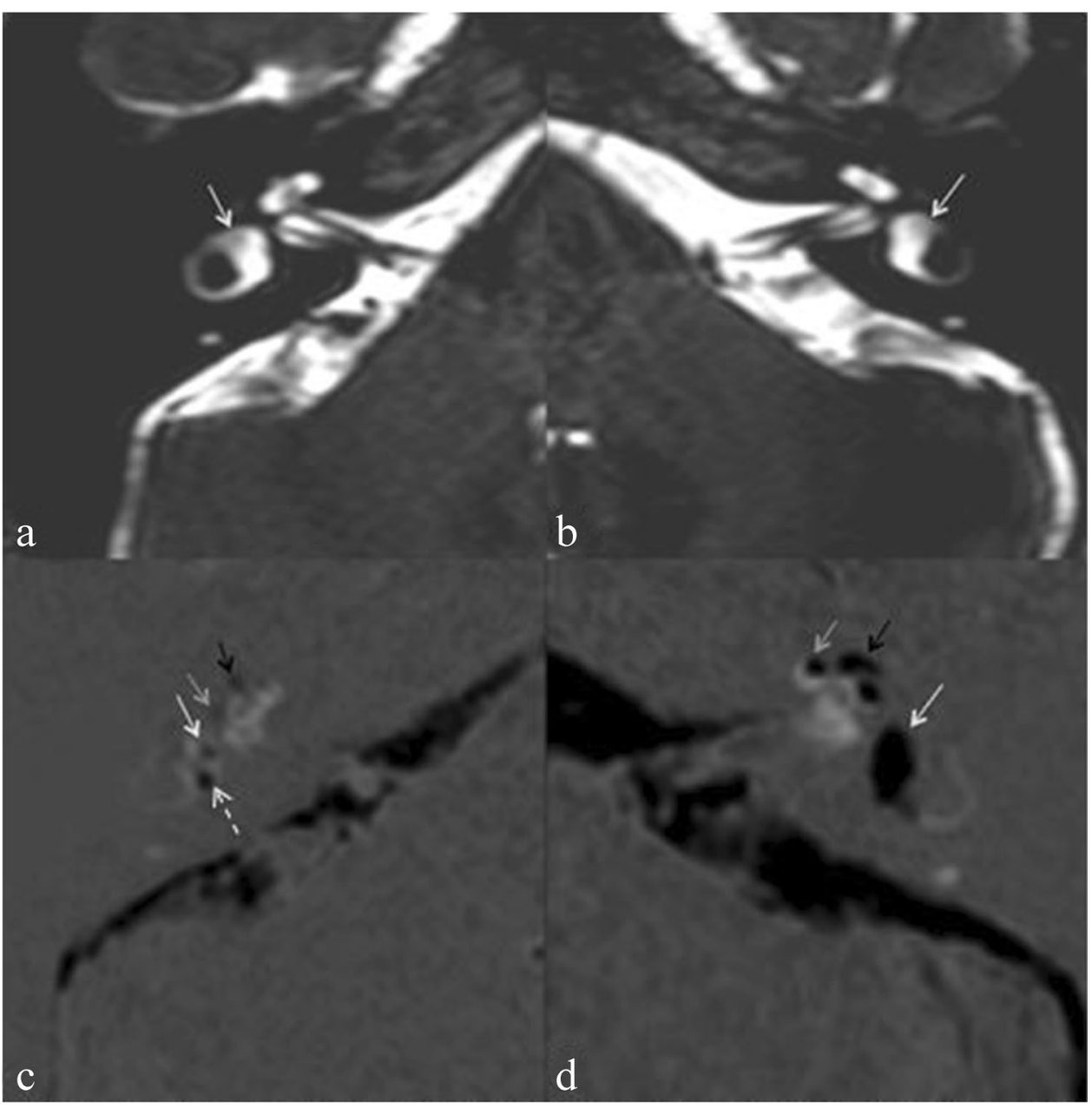

in proximity to the area to be studied, it is possible to improve the signal-to-noise ratio and the resolution of small surface coils without significantly increasing the acquisition time [48]. It is accepted that the greater the number of elements used, the greater the signal-to-noise ratio and the spatial resolution in the area close to the elements of the antenna (for example an antenna with 96 receiver channels proved to be superior to an antenna of 32 channels in terms of signal-to-noise ratio) [49]. However, a larger number of channels generate a huge influx of data 


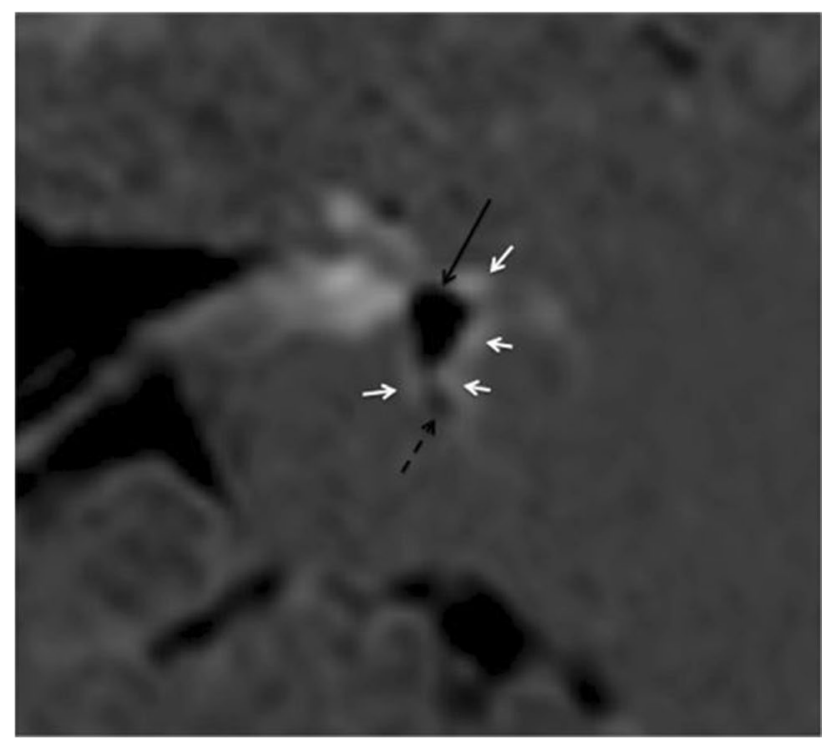

Fig. 7 Patient with clinical diagnosis of definite MD (recurrent spontaneous vertigo and left neurosensorial earing loss with 2 years of duration). Axial HYDROPS image showing non confluent enlargement of sacule (solid black arrow) and an utricle with normal size (dashed black arrow); perilymphatic space patent (white arrows)grade 1 endolymphatic hydrops according Bernaerts [49] (corresponding to B on Fig. 6), SURI > 1 [40]

and an overload on the image reconstruction computer, significantly increasing the reconstruction times. In the experience of Zou et al. [47], the best image quality of the

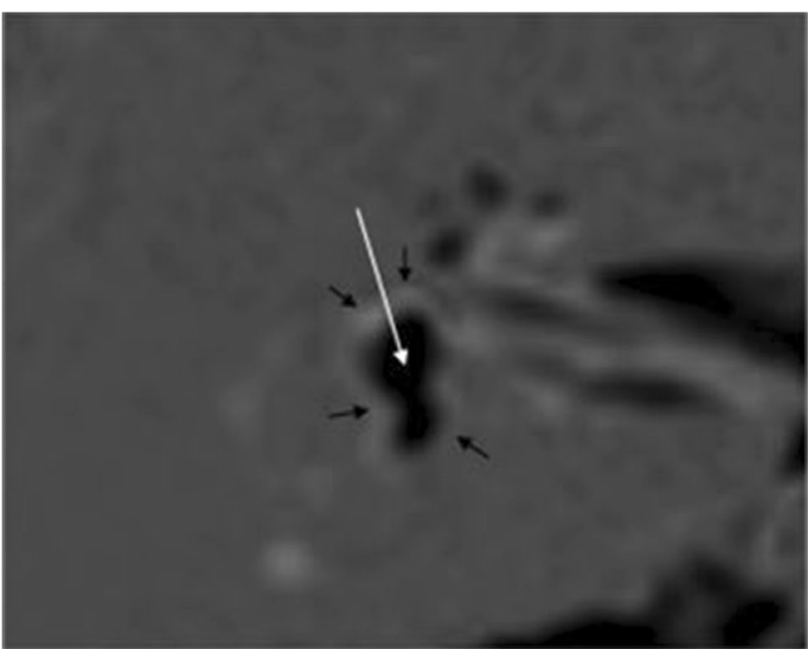

Fig. 8 Patient with diagnosis of definite MD (six years of duration of spontaneous recurrent vertigo and right neurosensorial earing loss). Axial MR HYDROPS images of right ear according lateral semicircular canal plane. Confluent enlargement of vestibular endolymphatic space (white arrow) incompletely effacing the perilymphatic space of the vestibule (black arrows)—grade 2 endolymphatic hydrops (Bernaerts)

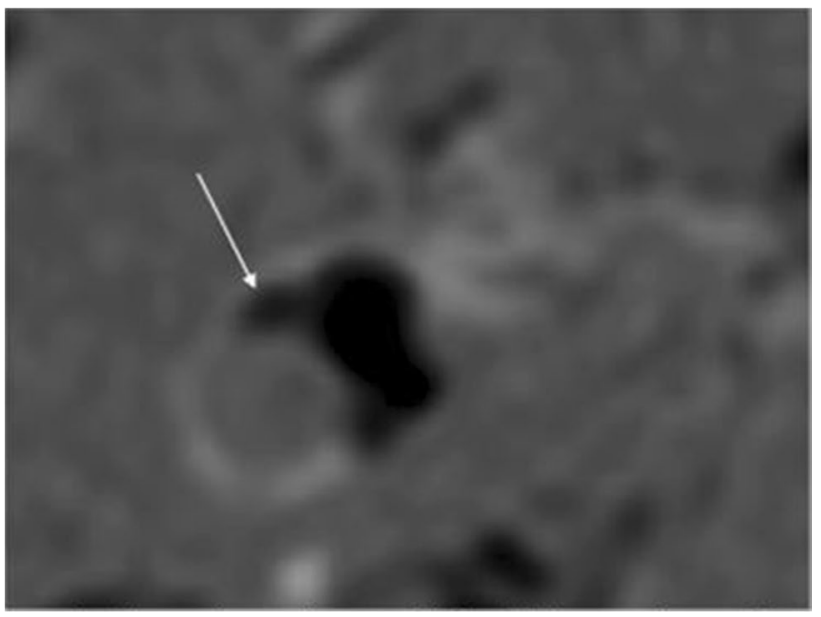

Fig. 9 Patient with recent diagnosis of definite MD (1 year duration of spontaneous recurrent vertigo and neurosensorial hearing loss) in the right ear. Axial MR images of right ear with HYDROPS showing vestibular endolymphatic hydrops completely obliterating the perilymphatic space of the vestibule with herniation into the ampullary region (white arrow)

inner ear is obtained with a combination of a 16-channel skull antenna and a $7 \mathrm{~cm}$ ear surface antenna. Small antennas have better spatial resolution and these surface coils are much closer to the ear than head coils; having a larger diameter allows obtaining signal from deeper structures,

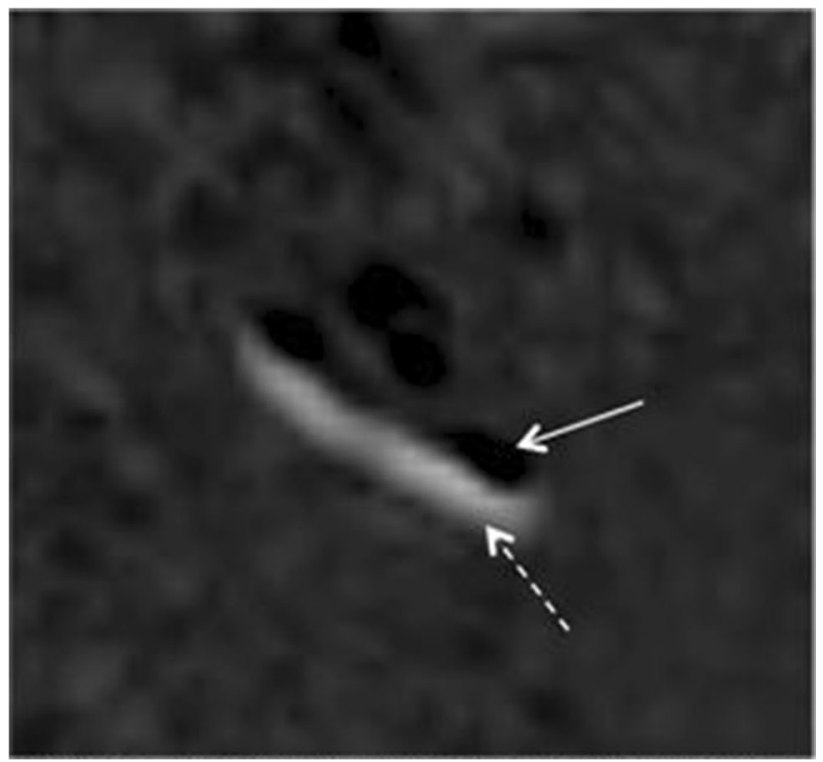

Fig. 10 Axial MR HYDROPS image of the left cochlea from a patient with fluctuating left earing loss and low frequency neurosensorial hypoacusia on audiogram. Grade 2 cochlear endolymphatic hydrops with complete effacement of the scala vestibuli (solid white arrow) and presence of contrast in the scala tympani (dashed white arrow) 


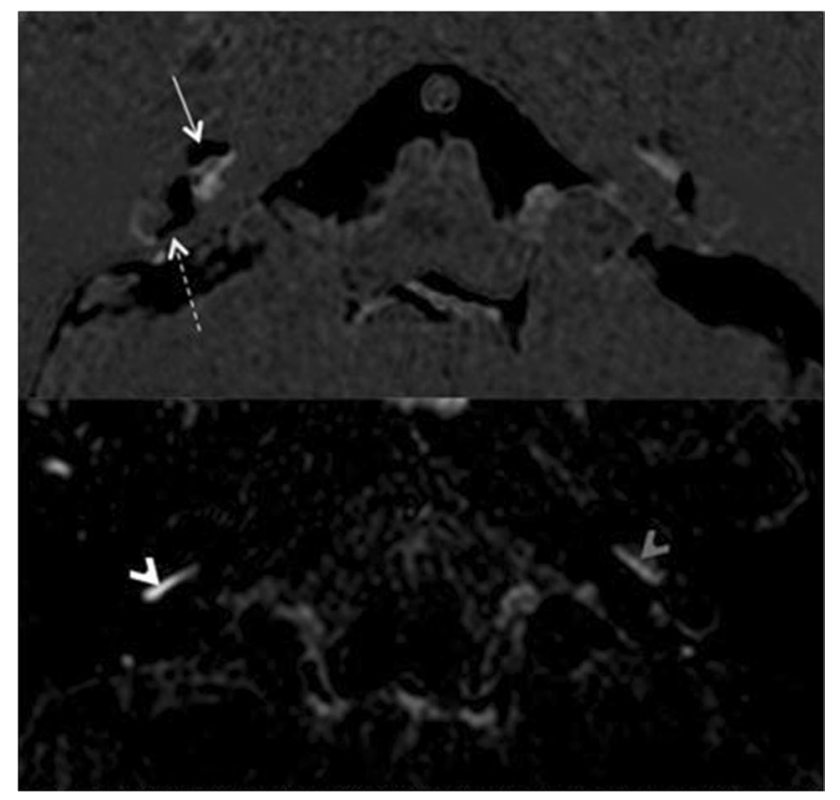

Fig. 11 Axial HYDROPS (above) and delayed 3D FLAIR after intravenous gadolinium injection (below) from a patient with definite right MD diagnosis. Grade 2 cochlear (solid white arrow) and grade 4 vestibular (dashed white arrow) hydrops is seen on the right ear; note utricle herniation into posterior limb of lateral semicircular canal (dashed white arrow). Asymmetric gadolinium enhancement is seen in perilymphatic space of basal turn of cochlea, pronounced on the right (white arrowhead), subtle on the left (grey arrowhead)

such as the inner ear, with a better signal-to-noise ratio than head antennas.

\section{Diagnostic imaging criteria}

Similar to the imaging technique, the imaging classification has also undergone several updates. The first semiquantitative scale of endolymphatic hydrops was developed in Nagoya in 2008 and published by Nakashima in 2009 [50], subdividing the cochlear and vestibular evaluation (since the volumes of the endolymphatic space in the vestibule and cochlea differ significantly in healthy subjects) (Table 2, Figs. 4 and 5). In the cochlea, this classification is based on the displacement of the Reissner membrane and on the proportional difference between the cochlear duct and the vestibular scala (grades 0 to 2) (Figs. 4, 6 and 10). In the vestibule, it consists on the ratio between the area of the endolymphatic space and the total area of the membranous vestibule (grades 0 to 2 ) when assessed in the axial plane. In Fig. 6(c), normal-sized endolymphatic spaces are seen-at the lower level of the vestibule, the saccule is usually a smaller round structure located anterior and medially; the utricle is larger than saccule and horizontally oriented in healthy ears and it is located at the most superior and posterior part of the vestibule (Fig. 1). According to this scale, several studies have found mild vestibular hydrops in 94-100\% of symptomatic ears in MD [42, 51-53], although it was also found in 53-100\% of asymptomatic ears [51-53], in $66 \%$ of other otological conditions [49], and in $90 \%$ of healthy volunteers [42]. It is therefore assumed that this cutoff value has low specificity but high sensitivity and can therefore be useful as an exclusion factor in MD when the clinical diagnosis is uncertain.

However, the cut-off value for hydrops varies between authors and Barath et al. [54] considers 50\% the cut-off value for vestibular hydrops grade 1 (Figs. 5C and 8), with grade 2 (Figs. 5D and 6d) being the maximum vestibular hydrops with practically total obliteration of the perilymphatic space. In order to avoid overdiagnosis of vestibular hydrops, Barath based on data from 20 asymptomatic individuals [55] in which the endolymphatic space could reach $41 \%$ of the total membranous labyrinth. Applying this scale, Barath misses endolymphatic hydrops in $10 \%$ of MD cases.

Gurkov introduces a 4-degree scale for evaluating endolymphatic hydrops to the original Nakashima scale by MRI (higher grade corresponding to extreme hydrops), correlating them for the first time with the audiovestibular function [56] (Figs. 5E, 9, 11, 12 and 13). This fourth stage corresponds to herniation of vestibular endolymphatic spaces anteriorly into de ampulla of lateral semicircular canal (Figs. 8 and 11), or posteriorly (utricle) into the (non ampullary) limb of lateral semicircular canal (Figs. 11 and 13).

In 2017, Attyè [42] reported an inversion of the saccule and utricle ratio (SURI) as the initial marker of the disease (SURI > 1 in 15/30 patients with MD disease and in none of the 30 healthy controls) (Figs. 5B and 7).

In 2019, new imaging criteria were proposed by the Antwerp group [57]—modified scale with 4 degrees (Figs. 4 and 5A-D ). The cochlear evaluation (Fig. 4) remains unchanged in relation to the Barath's criteria (Fig. 4) and a degree is added to the vestibular evaluation (Fig. 5B ): degree 0 - utricle and saccule occupying less than $50 \%$ of the total area of the membranous vestibule (Figs. 5A and 6C); grade 1-saccule equal to or greater than the utricle, but not confluent (Figs. 5B and 7); grade 2-confluent utricle and saccule, partially obliterating the perilymphatic space, with a rim of surrounding contrast enhancement left (Figs. 5C and 8); grade 3-maximum dilation of the utricle and saccule, confluent, with total obliteration of the perilymphatic space (Figs. 5D and 6D), and incipient herniation of the utricle to the non-ampullary aspect of the lateral semicircular canal (Figs. 11 and 13). In the same study, they also assessed the intensity of the gadolinium signal in the perilymphatic space as a measure of disruption of the hemato-perilymphatic barrier in the cochlea and vestibule in a semi-quantitative way, concluding that a higher signal intensity in the cochlear perilymphatic space is a very specific sign to the disease, and 


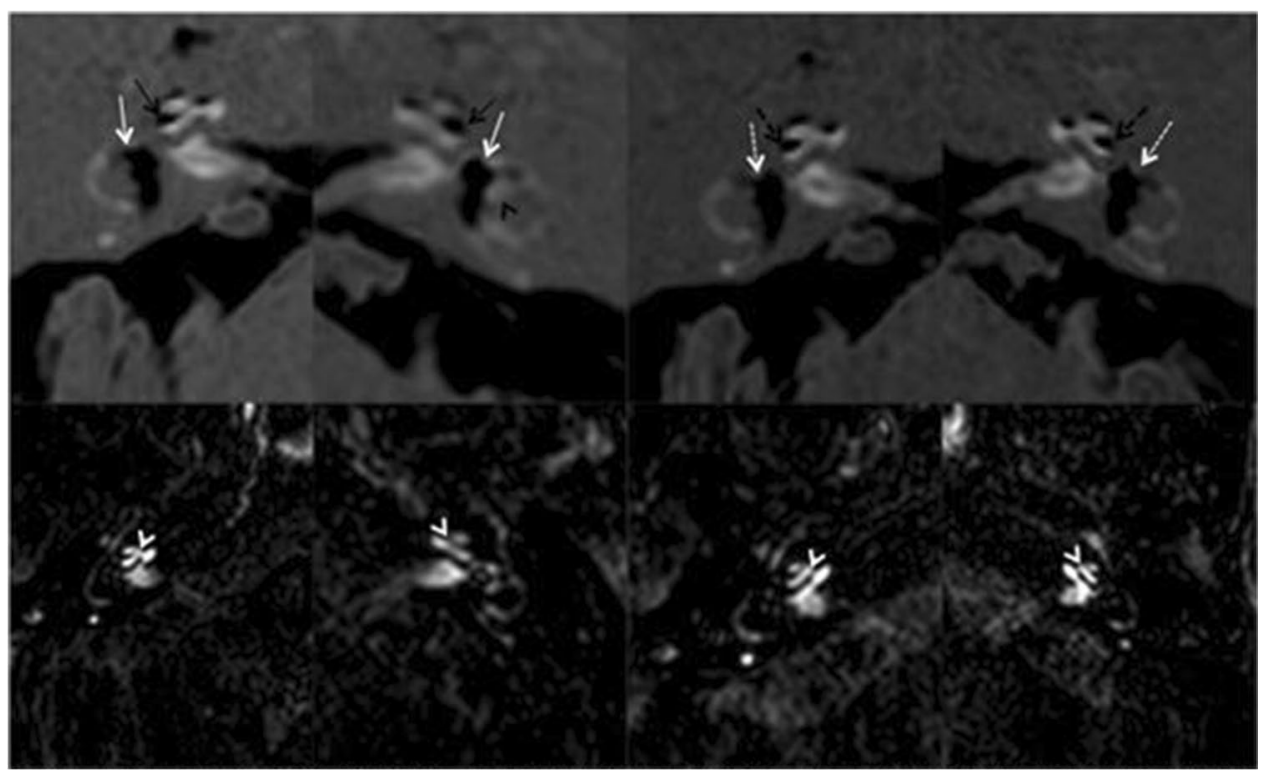

Fig. 12 Initial MRI (left images) and 2-year follow-up MRI (right images). Axial HYDROPS (above) and delayed T2 3D FLAIR $4 \mathrm{~h}$ after intravenous gadolinium injection (below) at midmodiolar level. Patient presents with low frequency bilateral neurosensorial earing loss (more severe on the right), spontaneous vertigo attacks lasting 5-10 min and fluctuating bilateral aural fullness, initially classified as atypical MD (cochlear MD) - MRI (left images) shows grade 2 bilateral cochlear hydrops (solid black arrows) and bilateral vestibular hydrops, grade 4 on the right and grade 2 on the left (solid white arrows); a rim of perilymphatic space is still patent in left ear (black arrowhead). Marked asymmetry of gadolinium enhancement in perilymphatic space of the cochlea (apical, middle, and basal turns), pronounced on the right,, subtle on the left (white arrowheads). In

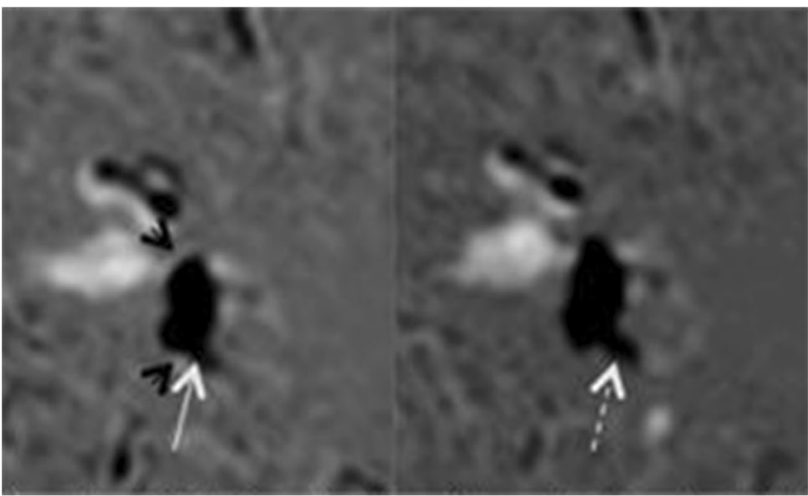

Fig. 13 Initial (left image) and 3-year follow-up (right image) axial HYDROPS images from a patient with diagnosis of left definite MD. On initial MRI vestibular, endolymphatic space is enlarged (solid white arrow), with confluence of utricle and saccule; perilymphatic space is partially effaced but a rim of perilymphatic space remains visible (black arrowheads). On follow-up MRI, an increase of vestibular endolymphatic space is seen, obliterating perilymphatic space, with herniation of utricle into posterior limb of lateral semicircular canal (dashed white arrow). Grade 2 cochlear hydrops remains stable
2 years (right images), the patient experienced progressive clinical worsening (increased in number of vertigo attacks, more than $30 \mathrm{~min}$ each, and worsening of earing loss, mainly on the left side), now fulfilling clinical criteria for definite MD. The right ear remains stable in follow-up MRI (grade 4 vestibular hydrops - dashed white arrow, grade 2 cochlear hydrops-back dashed arrow, and marked perilymphatic enhancement white arrowhead). The left ear shows clear clinical and imaging progression with vestibular hydrops changing from grade 2 to grade 4 (no perilymphatic space visible in left vestibuledashed white arrow, persistent grade 2 cochlear hydrops-dashed black arrow, and marked increase of perilymphatic enhancement in cochlea (now symmetric) — white arrowhead

therefore proposing a new evaluation algorithm, which starts with the signal intensity in the cochlear perilymphatic space (Fig. 11) and secondarily uses the 4-degree evaluation of the vestibule. This combined approach resulted in an improved diagnostic accuracy compared to previous grading systems. This important paper is retrospective, without an asymptomatic control group.

These relevant findings were corroborated in a recent paper from Steekelenburg [58]. In this study, the authors evaluated 220 patients with MR T2 3D and 3D FLAIR $4 \mathrm{~h}$ after intravenous gadolinium injection. These patients were retrospectively evaluated in terms of clinical characteristics and the evaluated ears were separated in 4 groups: definite $\mathrm{MD}$, probable MD, other vertigo-associated inner ear pathology not attributable to MD, and asymptomatic (contralateral MD ears). The images were scored for hydrops (Barath's scale and additional inversion saccule/utricle size) and visual signal intensity of the basal turn of the cochlea. Besides the retrospective design, the study hampers the potential to correlate imaging and clinical findings. It clearly points to an increased positive predictive value of the combination of endolymphatic hydrops and perilymphatic enhancement in 
Table 2 Classification of endolymphatic space by MRI from Nagoya described by Nakashima (2009) [47] and Barath [54]

\begin{tabular}{|c|c|c|c|c|}
\hline \multirow[t]{2}{*}{$\mathrm{EH}$ grade } & \multicolumn{2}{|l|}{ Vestibule } & \multicolumn{2}{|l|}{ Cochlea } \\
\hline & Nakashima & Barath & Nakashima & Barath \\
\hline No EH (grade 0) & $\leq 33 \%$ & $\leq 50 \%$ & No Reissner membrane dislocation & No dilated cochlear duct \\
\hline Light EH (grade 1) & $>33 \%, \leq 50 \%$ & $>50 \%,<100 \%$ & $\begin{array}{l}\text { Reissner membrane dislocation } \\
\text { Cochlear duct } \leq \text { scala vestibuli }\end{array}$ & Scala vestibuli partially obliterated \\
\hline Severe EH (grade 2) & $>50 \%$ & $100 \%$ & Cochlear duct $>$ scala vestibuli & Scala vestibuli totally obliterated \\
\hline
\end{tabular}

the diagnosis of definite MD in patients with suspected MD. This combination of imaging findings is distinctive for MD and is rarely present in other vertigo related entities. This can be particularly helpful in patients with atypical presentations of MD: endolymphatic hydrops and increased perilymphatic enhancement were found in $43 \%$ of patients of probable MD group; this can have great impact on treatment strategies for these patients although longitudinal research is needed to confirm clinical progression to definite MD.

For daily routine, we propose a systematic approach to conventional and endolymphatic hydrops imaging findings (Table 3). We would like to emphasize the importance of the correlation between clinical and radiological findings on patient management.

\section{Qualitative versus/(semi-) quantitative image evaluation}

The magnetic resonance technique of endolymphatic hydrops is based on volumetric sequences (T2 3D and T2 3D FLAIR) (Fig. 4), allowing multiplanar and three-dimensional reformatting and measurements of the structures.

The qualitative/semi quantitative evaluation method uses different planes to evaluate the vestibule and the cochlea.
Vestibule evaluation is classically described according to the plane of the lateral semicircular canal, using the lowest axial image at the inferior part of the vestibule. According to previous histological and imaging studies [58], the saccule occupies the most anterior, inferior, and medial part of the vestibule. Evaluating hydrops in the highest axial images through the vestibule can result in false positive diagnosis of vestibular hydrops. Cochlea evaluation is classically described according the axial plane along the greater axis of cochlear modiolus. The qualitative/semi quantitative method consists of a manual selection of areas of the vestibular endolymphatic space and its total area, thus estimating the ratio between the two, in order to classify the hydrops according to the scale adopted [54, 57, 59]. Some groups include the ampulla of the lateral semicircular canal on the measurements of the total area of the vestibule [12], which tends to facilitate the identification of anatomical structures; the ampulla inclusion tends to obtain lower ratios of endolymphatic space, as only extreme hydrops will produce herniation of the endolymph outside vestibule. Some publications omit the measurement method that was used. The evaluation of the cochlear space is more difficult, especially in the intravenous technique and with fewer channel antennas. It uses the axial plane along the greater axis of modiolus, also with human judgment factor inherent on the

Table 3 Methodology proposed for analysis and reporting conventional and endolymphatic hydrops imaging

\begin{tabular}{|c|c|c|}
\hline MRI sequences & Method & Purpose \\
\hline T2-weighted high resolution (isotropic) & Multiplanar analysis & $\begin{array}{l}\text { - Defining the outer margin of the perilymphatic space } \\
\text { - Defining the axial planes for vestibule and cochlea }\end{array}$ \\
\hline HYDROPS or delayed T2 FLAIR Gad & Multiplanar analysis & $\begin{array}{l}\text { - Identify saccule and utricle } \\
\text { - Grading vestibular hydrops according lateral semicircular } \\
\text { canal plane using the lowest slice } \\
\text { - Grading cochlear hydrops according midmodiolar plane and } \\
\text { using the maximum grade }\end{array}$ \\
\hline Delayed T2 FLAIR Gad & Signal intensity analysis & $\begin{array}{l}\text { - Look for asymmetric perilymphatic enhancement of endo- } \\
\text { lymphatic structures (mainly basal turn of the cochlea) }\end{array}$ \\
\hline $\begin{array}{l}\text { T1-weighted high-resolution pre and } \\
\text { post-gad (IAC) } \\
\text { T2-weighted high resolution (IAC) } \\
\text { T2 Flair and diffusion (brain) }\end{array}$ & $\begin{array}{l}\text { Evaluation of morphology and signal } \\
\text { of inner ear, cisterns, and brain } \\
\text { structures }\end{array}$ & $\begin{array}{l}\text { - Excluding other diagnosis (inner ear, cerebellar pontine } \\
\text { angle and brain) }\end{array}$ \\
\hline Report & & $\begin{array}{l}\text { Systematic } \\
\text { Radiological/clinical correlation }\end{array}$ \\
\hline
\end{tabular}


evaluation and with a lower rate of interobserver agreement. However, even with these drawbacks, interobserver agreement in the assessment of MRI of the cochlea and vestibule according Barath's scale [54] has proved to be higher than interobserver agreement in the clinical evaluation according to the current criteria for diagnosing MD [57]. Overall, we think radiologist expertise may play a crucial role on these high intra and inter observer agreement on imaging evaluation.

Adding to this, the evaluation of the enhancement of the perilymphatic enhancement has recently shown to be crucial on imaging evaluation of MD [57, 58] (Figs. 11 and 12). It consists on evaluation of signal intensity of basal turn of the cochlea and can be qualitative (visual inspection-perilymphatic enhancement asymmetry) or quantitative (signal intensity ratio of cochlear signal taking as a reference the signal intensity of brain structures such as middle cerebellar peduncle [58], cerebellar hemispheres [60], or medulla oblongata [61]). It might also serve as a marker of the degree of blood/perilymphatic barrier breakdown in some patients, a useful tool to follow-up patients with suspected or confirmed MD (Fig. 12).

Despite the high interobserver agreement on these semiquantitative techniques, the volume measurement of threedimensional structures is more objective, and can be automated [62, 63], but it needs good quality images, usually obtained with intratympanic technique. The volumetry may have an interesting role in the longitudinal assessment of patients and in monitoring the effect of therapies. Gurkov proposes a method of measuring volumes based on the combination of two sequences (T2 3D SPACE and T2 3D IR with intratympanic injection of gadolinium) and automated assessments of the total and relative volume between the different spaces [62]. In MD, Gurkov finds an average percentage value for the endolymphatic space of $15 \%$ in the cochlea and $28 \%$ in the vestibule.

The volume measurement technique was applied by a Japanese group [63] to 24 patients with chronic rhinosinusitis, without audiovestibular symptoms, with some additional data being obtained from a population theoretically without pathology. A recent study [64] made, for the first time, a semiautomatic volumetric assessment with $3 \mathrm{~T}$ MRI using intravenous technique, quantifying the volume of endolymphatic spaces and total membranous labyrinths in a population of 98 patients with MD disease and 47 healthy volunteers. This data, scarce in the literature, is of crucial importance, since it allows characterizing the "normal pattern" of the endolymphatic space in the absence of pathology. Healthy individuals were selected according to a questionnaire, with no history of hearing loss, vertigo, diseases of the middle or inner ear, cranial disease, cranial trauma, kidney disease, heart disease, medications, or gadolinium allergy. Unfortunately, the evaluation of the control group did not include audiometric (or vestibular) evaluation. They found percentages of endolymphatic hydrops of $13.9+/-7.9$ in the group of healthy volunteers and values significantly higher in symptomatic ears in MD disease, $24.1 \%$ for the cochlea and $37.5 \%$ for the vestibule, with the later findings consistent with those found by Gurkov [62]. This study is extremely important, although difficult to reproduce given the necessity of administration of contrast to individuals with no clinical indication for this. We must point out the absence of audiometric documentation of healthy individuals, which would have given an even greater credibility to MRI in MD.

Manual volumetric assessment takes a long time [65], despite being faster with semi-automated assessments [62]; this difference in the time of image evaluation is considered statistically significant in the study by Homann ( 2 min versus $14 \mathrm{~min}$ ) and the authors conclude that in daily clinical practice semi-quantitative evaluation is sufficient, using manual volumetry (cut to cut) in doubtful cases or following patients. However, they consider that in terms of the final result, volumetry is more accurate, especially if a semi-automatic method is available in the evaluation [62], in order to streamline the process and save time.

\section{Future perspectives of MRI}

It seems evident that MRI demonstration of endolymphatic hydrops has a significant correlation with clinical and physiological parameters and with the clinical course of the disease $[9,58,65-67]$. There is however a relative lack of data regarding healthy ears. Many studies consider the asymptomatic ear as the "normal" one but we know MD tends to be a bilateral process and endolymphatic hydrops can be identified in the pre-symptomatic phase of the disease, so it is crucial to characterize the membranous labyrinth in healthy individuals with documented normal audiograms and establish normal patters in the healthy population, in order to serve as a comparison with inner ear disorders. We suggest studying patients who need contrast MRI of other anatomical regions, without audiovestibular symptoms and with normal audiogram, for example, patients with failed back surgery (disc herniation).

We know that MD is a chronic condition but the natural history of the disease is not well elucidated and longitudinal studies regarding evolution of endolymphatic spaces in MD are scarse [68-71]. Although the scientific evidence is poor, it seems some patients have progression of endolymphatic hydrops in the course of MD (Fig. 13). Besides that, there are still few controlled clinical studies on the effectiveness of different treatments in MD and there is currently no treatment with proven benefit. No benefit was seen on endolymphatic hydrops in patients under medical 
treatment $[68,70]$, although factors as insufficient dosage, insufficient MRI resolution, and short follow-up period have been implicated. These findings are not supported by a longitudinal study with acetazolamide therapy [70] (5 patients) that points to a clinical and imaging improvement in majority of patients, with one clinical and imaging recurrence with treatment interruption. Surgical endolymphatic sac decompression (7 patients) seems to result in clinical and imaging improvement in a 2-year followup study. The ideal treatment for MD should reverse the progression of endolymphatic hydrops, the pathological substrate of the disease. Volumetric evaluation of the endolymphatic space [62] may play a central role as a biomarker for response in MD as it permits quantitative assessment of the effect of distinct therapeutic strategies on endolymphatic hydrops.

\section{Conclusion}

MD diagnostic criteria remain clinical although endolymphatic hydrops imaging seems more reproducible than clinical evaluation. Despite the longstanding recognition of endolymphatic hydrops as a central pathophysiological factor of the disease and scientific evidence of a correlation between endolymphatic hydrops and clinical parameters, MRI identification of endolymphatic hydrops is currently not considered in the diagnosis of the disease and this is particularly relevant in management of monosymptomatic, incomplete phenotypes, and bilateral clinical presentations of MD.

Improvement of MRI technique, recognition of imaging pitfalls, and keeping regular clinical feedback is important. Different study protocols should be adjusted to each MRI scanner, according to their specificities, and it is important that each center establishes the standard of normality for the applied technique; obtaining data for healthy individuals is, however, hampered by ethical issues related to the administration of contrast in healthy individuals. Volumetric assessment of endolymphatic spaces may be helpful in assessment of the efficacy of different treatment strategies.

Longitudinal hydrops studies are particularly scarce and those can bring some light to the pathophysiology of MD.

Funding No funding was received for this study.

\section{Declarations}

Conflicts of interest The authors declare that they have no conflict of interest.

Ethical approval All procedures performed in the studies involving human participants were in accordance with the ethical standards of the institutional and/or national research committee and with the 1964 Helsinki Declaration and its later amendments or comparable ethical standards.

Informed consent Informed consent was obtained from all individual participants included in the study.

\section{References}

1. Havia M, Kentala E, Pykko I (2005) Prevalence of Menière's disease in general population of Southern Finland. Otolaryngol Head Neck Surg 133(5):762-768

2. Hallpike CS, Cairns H (1938) Observations on the pathology of the Menière's syndrome. Pro R Soc Med 31:1317-1336

3. Yamakawa K (1938) Uber die pathologische Veranderung bei einem Menière-kranken. Procedings of 42nd Annual Meeting Oto-Rhino-Laryngol Soc Japan 4: 2310-2312.

4. Hinchcliffe R (1967) An attempt to classify the primary vertigos. J Laryngol Otol 81:849-857

5. Hinchcliffe R (1973) Menière's syndrome. Recent advances in otolaryngology. In: Ransome J, Holden H, Bull TR (eds). Churchill Livingstone, Edinburgh. Vol 12, pp127-143.

6. Committee on Hearing and Equilibrium (1972) Report of Subcommittee on Equilibrium and its Measurement. Menière's disease: criteria for diagnosis and evaluation of therapy for reporting. Trans Am Acad Ophtalmol Otolaryngol 76:1462-1464

7. Pearson BW, Brackmann DE (1985) Committee on Hearing and Equilibrium guidelines for reporting treatment results in Menière's disease. Otolaryngol Head Neck Surg 93(5):579-581

8. AAO-HNS (1995) Committee on earing and equilibrium guidelines for the diagnosis and evaluation of therapy in Menière's disease. American Academy of Otolangology Head and Neck Foundation, Inc. Otolaryngol Head Neck Surg 113(3); 181-185.

9. Pykko I, Nakashima T, Yoshida T, Zou J, Naganawa S (2013) Menière's disease: a reappraisal supported by a variable latency of symptoms and the MRI visualization of endolymphatic hydrops. BMJ Open 3(2). https://doi.org/10.1136/bmjopen-2012-001555.

10. Lopez-Escamez JA, Carey J, Chung WH, Goebel JA, Magnussen M, Mandala M et al (2015) Diagnostic criteria for Menière's Disease. J Vestib Res 25(1):1-7

11. Nakashima T, Pykko I, Poe D (2016) New outlook on Menière's disease. Nat Rev Dis Primers. https://doi.org/10.1038/nrdp.2016. 28

12. Gurkov R, Pykko I, Zou J, Kentala E (2016) What is Menière's disease? A contemporary re-evaluation of endolymphatic hydrops. J Neurol 263(Suppl 1):S71-S81

13. Gurkov R, Hornibrook J (2018) On the classification of hydropic ear disease (Menière's disease). HNO 66(6):455-463

14. Gurkov R, Jerin C, Flatz W, Maxwell R (2019) Clinical manifestations of hydropic ear disease (Ménière's). Eur Arch Otolaryngol 276(1):27-40

15. Foster CA, Breeze RE (2013) Endolymphatic hydrops in Menière's disease: cause, consequence, or epiphenomenon? Otol Neurotol 34(7):1210-1214

16. Salt AN, Plontke SK (2010) Endolymphatic hydrops: pathophysiology and experimental models. Otolaryngol Clin North Am 43:971-983

17. Weissman JL (1997) Imaging of Meniere's disease. Otolaryngol Clin North Am 30(6):1105.16

18. Zou J, Pyykko I, Bjelke B, Dastidar P, Toppil E (2005) Communication between the perilymphatic scalae and spiral ligament visualized by in vivo MRI. Audiol Neurotol 10(3):145-152 
19. Nakashima T, Naganawa S, Sugiura M, Teranishi M, Sone M, Hayashi $\mathrm{H}$ et al (2007) Visualization of endolymphatic hydrops in patients with Menière's disease. Laryngoscope 117(3):415-420

20. Naganawa S, Sigiura M, Kawamura M, Fakatsu H, Sone M, Nakashima T (2008) Imaging of endolymphatic and perylymphatic fluid at $3 \mathrm{~T}$ after intratympanic administration of gadolinium-diethylene-triamine pentaacetic acid. AJNR 29(4):724-726

21. Naganawa S, Satake H, Hawamura M, Fakatsu H, Sone M, Nakashima T (2008) Separate visualization of endolymphatic space, perilymphatic space and bone by a single sequence; $3 \mathrm{D}$-inversion recovery imaging utilizing real reconstruction after intratympanic Gd-DTPA administration at 3 Tesla. Eur Radiol 18(5):920-924

22. Jerin C, Krause E, Ertl-Wagner B, Gurkov R (2014) Longitudinal assessment of endolymphatic hydrops with contrast-enhanced magnetic resonance imaging of the labyrinth. Otol Neurotol 35(5):880-883

23. Nakashima T, Naganawa $S$, Theranishi M, Tagaya M, Nakata S, Sone M et al (2010) Endolymphatic hydrops revealed by intravenous gadolinium injection in patients with Ménière's disease. Acta Otolaryngol 130(3):338-343

24. Fiorino F, Pizzini FB, Beltramello A, Mattellini B, Barbieri F (2011) Reliability of magnetic resonance imaging performed after intratympanic administration of gadolinium in the identification of endolymphatic hydrops in patients with Ménière's disease. Otol Neurotol 32(3):472-477

25. Gu X, Fang ZM, Liu Y, Wang ZW, Zhang R, Chen X (2015) Diagnostic advantages of intratympanically gadolinium contrastenhanced magnetic resonance imaging in patients with bilateral Ménière's disease. Am J Otolaryngol 36:67-73

26. Pykko I, Zou J, Poe D, Nakashima T, Naganawa S (2010) Magnetic resonance imaging of the inner ear in Ménière's disease. Otolaryngol Clin North Am 43(5):1059-1080

27. Louza J, Krause E, Gurkov R (2013) Audiologic evaluation of Ménière's disease patients one day and one week after intratympanic application of gadolinium contrast agent: our experience in sixty-five patients. Clin Otolaryngol 38(3):262-266

28. Louza J, Krause E, Gurkov R (2015) Hearing function after intratympanic application of gadolinium-based contrast agent: a long term evaluation. Laryngoscope 125(10):2366-2370

29. Louza J, Flatz W, Krause E, Gurkov R (2012) Short term audiologic effect of intratympanic gadolinium contrast agent application in patients with Menière's disease. Am J Otolaryngol 33(5):533-537

30. Iida T, Teranishi M, Yoshida T, Otake H, Sone M, Kato M et al (2013) Magnetic resonance imaging of the inner ear after both intratympanic and intravenous gadolinium injections. Acta Otolaryngol 133(5):434-438

31. Naganawa S, Yamazaki M, Kawai H, Bokura K, Sone M, Nakashima T (2010) Visualisation of endolymphatic hydrops in Menière's disease with single dose intravenous gadolinium-based contrast media using heavily T2-weighted 3D FLAIR. Magn Res Sci 9:237-242

32. Cho SY, Ahn JM, Choi JE, Park HW, Kim YK, Kim HJ et al (2018) Usefulness of intravenous gadolinium inner ear mr imaging in diagnosis of Menière's disease. Sci Rep 8(1):17562. https:// doi.org/10.1038/s41598-018-35709-5

33. Gurkov R, Todt I, Jadeed R, Sudhoff H, Gehl HB (2020) Laterality of audiovestibular symptoms predicts laterality of endolymphatic hydrops in hydropic ear disease (Meniere). Otol Neurotol 41(9):e1140-e1144

34. Venkatasamy A, Veillon F, Fleury A, Eliezer M, Eid MA, Romain $B$ et al (2017) Imaging of the saccule for the diagnosis of endolymphatic hydrops in Ménière disease, using a three-dimensional T2-weighted steady state free precession sequence : accurate, fast, and without contrast material intravenous injection. Eur Radiol Exp 1(1):14. https://doi.org/10.1186/s41747.017-0020-7

35. Dominguez P, Naganawa (2008) Letter to the editor on the article: "Saccular measurements in routine MRI can predict hydrops in Ménière's disease", by Simon F et al. Eur Arch Otorhinolaryngol 275(1):311-2

36. Naganawa S, Susuki K, Yamazaki M, Sakurai Y, Ikeda M (2014) Time course for measuring endolymphatic size in healthy volunteers following intravenous administration of gadoteridol. Magn Reson Med Sci 13:73-80

37. Naganawa S, Yamasaki M, Kawai H, Bokura K, Sone M, Nakashima T (2012) Imaging of Menière's disease after intravenous administration of single-dose gadodiamide: utility of subtraction images with different inversion time. Magn Reson Med Sci 11(3):213-219

38. Naganawa S, Yamazaki M, Kawai H, Bokura K, Sone M, Nakashima T (2013) Imaging of Menière's disease after intravenous administration of single-dose of gadodiamide: utility of multiplication of MR cisternography and HYDROPS image. Magn Reson Med Sci 12(1):63-68

39. Sepahdari AR, Ishiyama G, Vorasubin N, Peng KA, Linetsky M, Ishiyama A (2015) Delayed intravenous contrast enhanced 3D FLAIR MRI in Menière's disease: correlation of quantitative measures of endolymphatic hydrops with hearing. Clin Imaging 39(1):26-31

40. Connor SEJ, Pai I (2020) Endolymphatic hydrops magnetic resonance imaging in Ménière's disease. Clin Radiol Sep 3;S0009-9260(20)30307-X. https://doi.org/10.1016/j.crad.2020. 07.021 .

41. Elizier M, Gillibert A, Tropres I et al (2017) Influence of inversion time on endolymphatic evaluation in 3D-FLAIR imaging. $\mathrm{J}$ Neuroradiol 44:339-343

42. Attyé A, Eliezer M, Boudiaf N, Tropres I, Chechin D, Schmerber S et al (2017) MRI of endolymphatic hydrops in patients with Ménière's disease: a case-controlled study with a simplified classification based on saccular morphology. Eur Radiol 27(8):3138-3146

43. Naganawa S, Yamazaki M, Kawai H, et al (2012) Visualization of endolymphatic hydrops in Ménière's disease after single dose intravenous gadolinium-based contrast medium: timing of optimal enhancement. Magn Reason Med Sci 11:43e51.

44. Grieve S, Obholzer R, Malitz N, Gibson W, Parker G (2012) Imaging of endolymphatic hydrops in Meniere's disease at $1.5 \mathrm{~T}$ using phase-sensitive inversion recovery: (1) demonstration of feasibility and (2) overcoming the limitations of variable gadolinium absorption. Eur J Radiol 81(2):331-8.

45. Naganawa S, Yamazaki M, Kawai H, Bokura K, Sone M, Nakashima T (2013) Visualization of endolymphatic hydrops in Ménière's disease after intravenous administration of single-dose gadodiamide at 1.5T. Magn Reson Med Sci 12(2): 137-9.

46. Gurkov R, Lutsenko V, Babkina T, Valchyshyn S, Situkho M (2021) Clinical high-resolution imaging and grading of endolymphatic hydrops in Hydropic Ear Disease at $1.5 \mathrm{~T}$ using the two-slice grading for vestibular endolymphatic hydrops in less than $10 \mathrm{~min}$. Eur Arch Oto-Rhino-Laryngol: https://doi.org/10. 1007/s00405-021-06731-7.

47. Zou J, Pykko I, Yoshida T, Gurkov R, Shi H, Li Y et al (2015) Milestone research on Ménière's disease by visualizing endolymphatic hydrops using gadolinium-enhanced inner ear MRI and the challenges in clinical applications. Austin J Radiol 2(6):1035

48. Roemer PB, Edelstein WA, Hayes CE, Souza SP, Mueller OM (1990) The NMR phase array. Magn Reson Med 16:192-225

49. Wiggins GC, Polimeni JR, Pottahast A, Schmitt M, Alagappan V, Wald LL (2009) 96-Channel receive-only head coil for 3 Tesla: design optimization and evaluation. Magn Reson Med 62:754-762 
50. Nakashima T, Naganawa S, Pykko I, Gibson W, Sone M, Nakata S, Teranishi M (2009) Grading Endolymphatic Hydrops using Magnetic Resonance Imaging. Acta Otolaryngol Suppl 580:5-8

51. Sano R, Teranishi M, Yamazaki M et al (2012) Contrast enhancement of the inner ear in magnetic resonance images taken 10 minutes or 4 hours after intravenous gadolinium injection. Acta Otolaryngol 132:241-246

52. Yoshida T, Sugimoto S, Teranishi M, Otake H, Yamazaki M, Naganawa $S$ et al (2018) Imaging of the endolymphatic space in patients with Menière's disease. Auris Nasus Larynx 45(1):33-38

53. Tagaya M, Yamazaki M, Teranishi M, Naganawa S, Yoshida T, Otake H et al (2011) Endolymphatic hydrops and blood-labyrinth barrier in Menière's disease. Acta Otolaryngol 131(5):474-479

54. Barath K, Schuknecht B, Naldi AM, Schrepfer T, Bockisch CJ, Hegemann SC (2014) Detection and grading of endolymphatic hydrops in Ménière's disease using MR imaging. AJNR Am J Neuroradiol 35(7): 1387-1392

55. Liu F, Huang W, Meng X, Wang Z, Liu X, Chen Q (2012) Comparison of non invasive evaluation of endolymphatic hydrops in Menière's disease and endolymphatic space in healthy volunteers using magnetic resonance imaging. Acta Otolaryngol 132(3):234-240

56. Gurkov R, Flatz W, Louza J, Strupp M, Krause E (2011) In vivo visualization of endolymphatic hydrops in patients with Ménière's disease: correlation with audiovestibular function. Eur Arch Otolaryngol 268:1743-1748

57. Bernaerts A, Vanspauwen R, Blaivie C, van Dinther J, Zarowski A, Wuyts FL et al (2019) The value of fours stage vestibular hydrops grading and asymmetric perilymphatic enhancement in the diagnosis of Menière's disease on MRI. Neuroradiology 61(4):421-429

58. Stekelenburg JM, Weijnen A, Pont LMH, Vijbrief OD, Bommelié CC, Koopman JP, Verbist BM, Blom HM, Hammer S (2020) Value of endolymphatic hydrops and perilymph signal intensity in suspected Menière disease. AJNR Am J Neuroradiol 41(3):529-534

59. Gurkov R, Flatz W, Louza J, Strupp M, Ertl-Wagner B, Krause E (2012) In vivo visualized endolymphatic hydrops and inner ear functions in patients with electrococleographically confirmed Menière's disease. Otol Neurotol 33(6):1040-1045

60. Shi S, Guo P, Wang W (2018) Magnetic resonance imaging of Ménière's disease after intravenous administration of gadolinium. Ann Otol Rhinol Laryngol 127:777-782

61. Tagaya M, Yamazaki M, Teranishi M et al (2011) Endolymphatic hydrops and blood-labyrinth barrier in Ménière's disease. Acta Otolaryngol 131:474-479
62. Gurkov R, Berman A, Dietrich O, Flatz W, Gerin C, Krause E et al (2015) MR volumetric assessment of endolymphatic hydrops. Eur Radiology 25(2):585-595

63. Inui H, Sakamoto T, Ito T, Kitahara T (2016) Magnetic resonance volumetric measurement of endolymphatic space in patients without vertiginous or cochlear symptoms. Acta Otolaryngol 136(12):1206-1212

64. Ito T, Inui H, Miyasaka T, Shiozaki T, Hasukawa A, Yamanaka T et al (2019) Endolymphatic volume in patients with Ménière's disease and healthy controls: three-dimensional analysis with magnetic resonance imaging. Laryngoscope Investig Otolaryngol 4(6):653-658

65. Homann G, Vieth V, Weiss D, Nikolaou K, Heindel W, Notohamiprodjo $M$ et al (2015) Semi-quantitative vs volumetric determination of endolymphatic space in Menière's disease using endolymphatic hydrops 3T-HR-MRI after intravenous gadolinium injection. PLoS ONE 10(3):e0120357. https://doi.org/10.1371/ journal.pone. 0120357

66. Gurkov R, Flatz W, Louza J, Strupp M, Krause E (2011) In vivo visualisation of endolymphatic hydrops in patients with Ménière's disease: correlation with audiovestibular function. Eur Arch Otorhinolaryngol 268(12):1743-1748

67. Gurkov R, Flatz W, Ertl-Wagner B, Krause E (2012) In vivo visualised endolymphatic hydrops and inner ear functions in patients with electrococleographically confirmed Ménière's disease. Otol Neurotol 33(6):1040-1045

68. Gurkov R, Flatz W, Keeser D, Strupp M, Ertl-Wagner B, Krause E (2013) Effect of standard-dose Betahistine on endolymphatic hydrops: an MRI pilot study. Eur Arch Otorhinolaryngol 270(4):1231-1235

69. Liu F, Huang W, Chen Q, Meng X, Wang Z, He Y (2014) Non invasive evaluation of the effect of endolymphatic sac decompression in Meniere's disease using magnetic resonance imaging. Acta Otolaryngol 134(7):666-671

70. Sepahdari A, Vorasubin N, Ishiyama G, Ishiyama A (2016) Endolymphatic hydrops reversal following acetazolamide therapy: demonstration with delayed intravenous contrast-enhanced $3 \mathrm{D}$ FLAIR MRI. AJNR 37(1):151-154

71. Fukushima M, Kitahara T, Oya R, Akahani S, Inohara H, Naganawa S, Takeda N (2017) Longitudinal up-regulation of endolymphatic hydrops in patients with Meniere's disease during medical treatment. Laryngoscope Investig Otolaryngol 2(6):344-350

Publisher's note Springer Nature remains neutral with regard to jurisdictional claims in published maps and institutional affiliations. 